\title{
Is there a second critical point in liquid water?
}

\author{
H.E. Stanley ${ }^{a}$, C.A. Angell ${ }^{b}$, U. Essmann ${ }^{a, 1}$, M. Hemmati ${ }^{b}$, \\ P.H. Poole ${ }^{\mathrm{a}, \mathrm{b}}$ and F. Sciortino ${ }^{\mathrm{a}, \mathrm{c}}$ \\ a Center for Polymer Studies and Department of Physics, Boston University, \\ Boston, MA 02215, USA \\ b Department of Chemistry, Arizona State University, Tempe AZ 85287, USA \\ " Dipartimento di Fisica, Università di Roma "La Sapienza", Piazzale Aldo Moro, \\ 00185 Roma, Italy
}

\begin{abstract}
The supercooled and stretched regions of the phase diagram of simulated liquid water are investigated by calculating the equation of state of the ST2 and TIP4P pair-potentials. We find that simulated water does not display a re-entrant spinodal and that the projection of the density maximum line in the plane of pressure and temperature becomes positively sloped on stretching. The well-known anomalous behavior of supercooled water is tentatively associated with the existence of an inaccessible critical point. Evidence is presented that suggests the association of this new critical point with the transition between low density and high density amorphous solid water. We show how the observed transformation behavior of the two forms of amorphous solid water can be explained in terms of a first order phase transition, via a consideration of the limits of metastability associated with this kind of transition, and support this interpretation with simulations of the amorphous solid. We therefore propose a phase diagram which accounts for the behavior of both liquid and amorphous solid water.
\end{abstract}

\section{Introduction}

Liquid water is one of the most studied complex fluids. The possibility for the water molecule to form hydrogen bonds and to create an open network in both the solid and the liquid phases is at the basis of its peculiar behavior (figs. 1 and 2). The inverse correlation between the energy and the volume at a microscopic level, due to the strength and directionality of the hydrogen bond, is reflected in the anomalous behavior of the liquid state. In the supercooled and the stretched regions of the phase diagram, the well-known anomalies of liquid water become more pronounced $[2,3]$. The effects of the hydrogen bond network become more and more important with decreasing temperature

1 Present address: Department of Chemistry, University of North Carolina, Chapel Hill NC 27599, USA. 


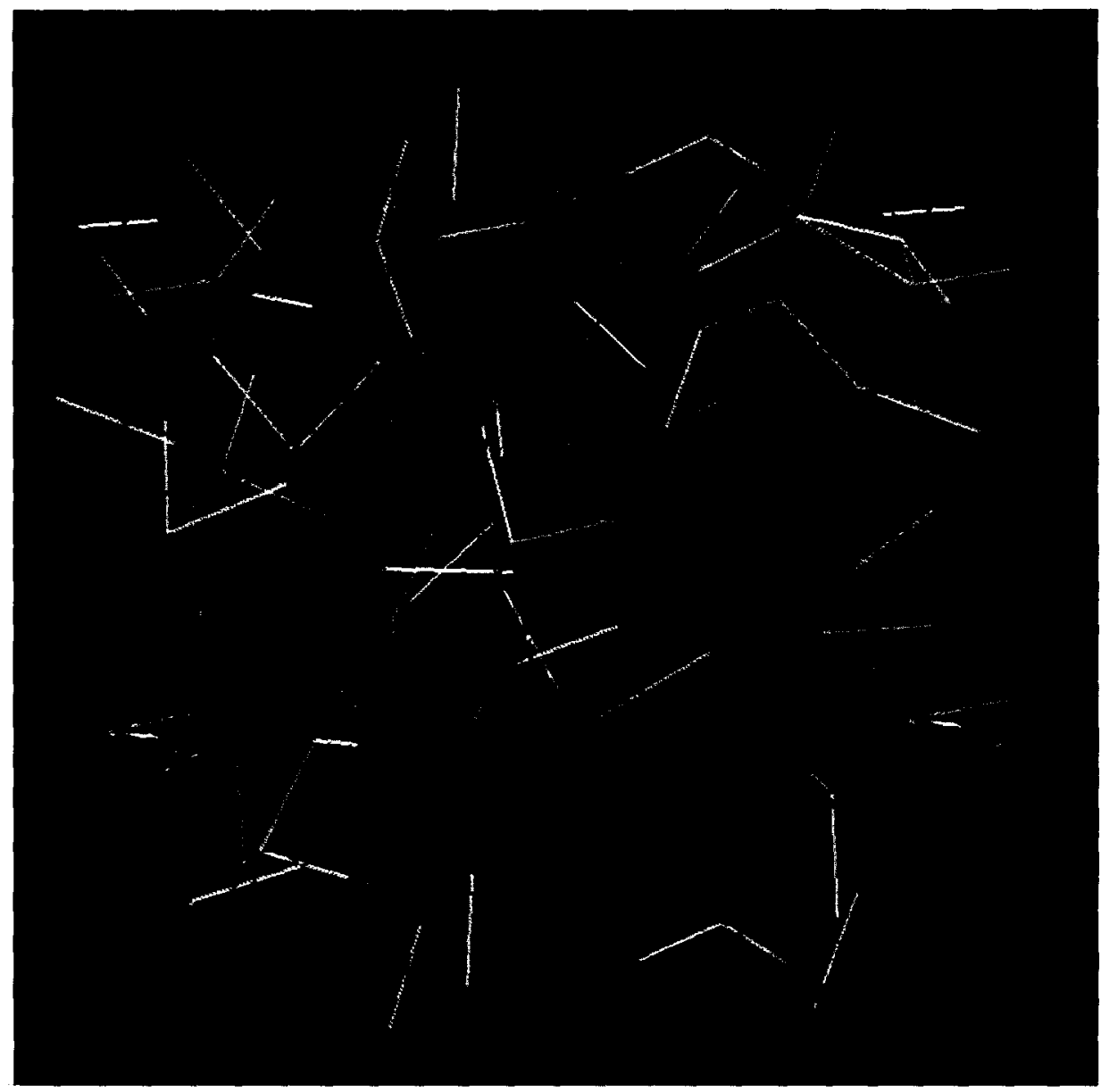

Fig. 1. The hydrogen bond network in a simulation of 216 water molecules is here visualized by showing not the molecules themselves, but the bonds between them. The colors represent the energy of the bonds, blue indicating the strongest bonds (i.e. most negative interaction energy) and red the weakest (i.e. least negative interaction energy). Courtesy of $P$. Trunfio.

(lowering of the local energy) as well as with increasing negative pressure (increasing of the local volume) [4]. In this article we give a report of a study on the phase behavior of metastable water based on analyses of extensive molecular dynamics simulations with water-water potentials that mimic the anomalous behavior of the real liquid.

The well-known equilibrium phase diagram of water is shown in fig. 3. In the dashed area the liquid state is stable. When the equilibrium solid-liquid or liquid-gas lines are crossed, the liquid enters into a thermodynamic state that is metastable compared to the solid or to the gas. The liquid can be supercooled 


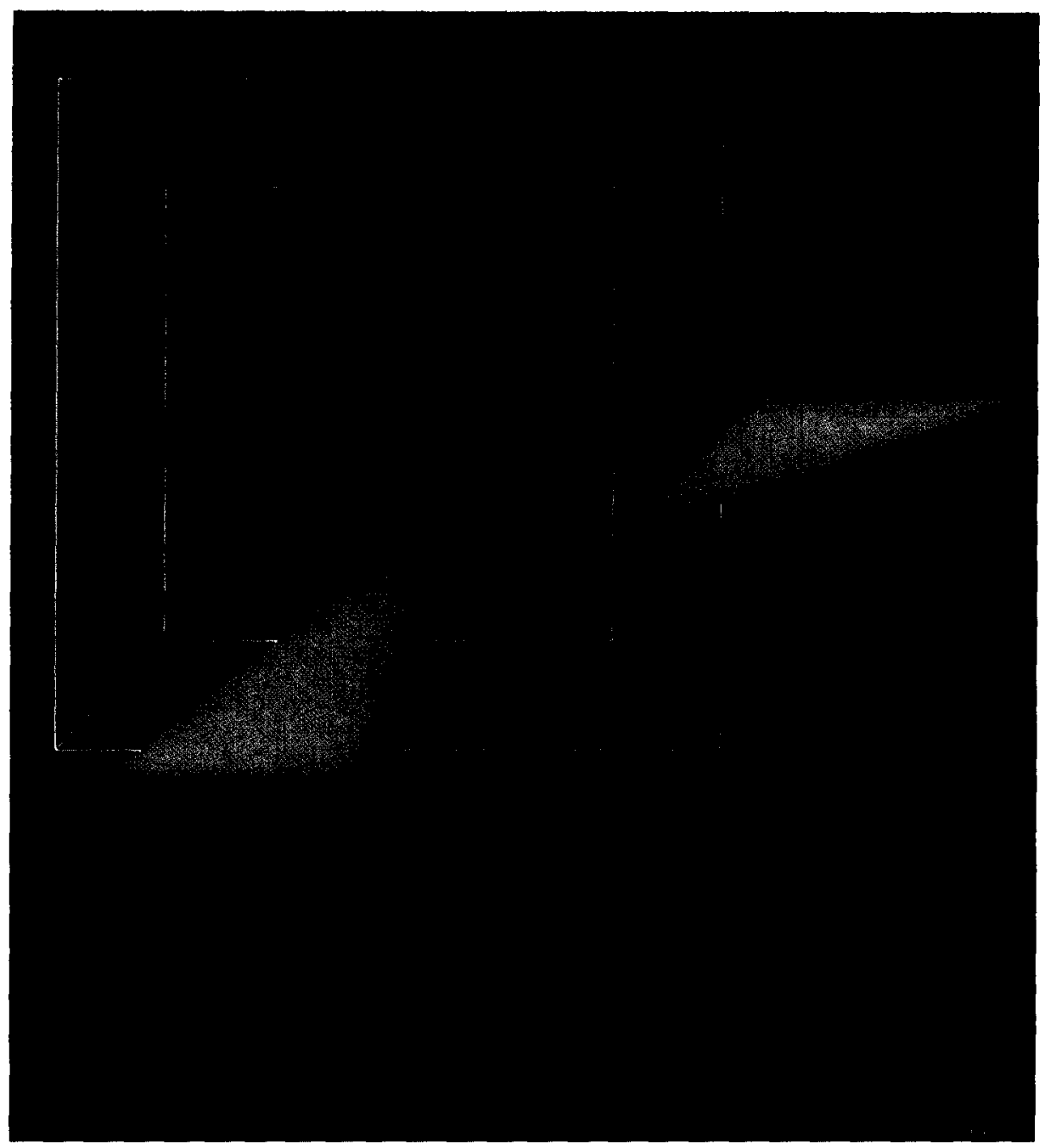

Fig. 2. The local environment around a particular water molecule strongly effects its behavior [1]. For example, shown here are two sub-regions of a simulation of water. In one, the central molecule is surrounded by four tetrahedrally placed neighbors; this kind of environment promotes strong hydrogen bonding, and hence has a low energy, low mobility, and low local density. In the other, the environment of the central molecule contains five neighbors; in this case the hydrogen bonds are imperfect, leading to higher energy, higher mobility, and higher local density. By courtesy of B. Ostrovsky.

or stretched only to a finite extent. Then, even in very clean samples, internal fluctuations drive the system from the metastable liquid state to the stable solid or gas phase, imposing a limit on the range of thermodynamic states accessible to experiments. These limits of supercooling and stretching define 


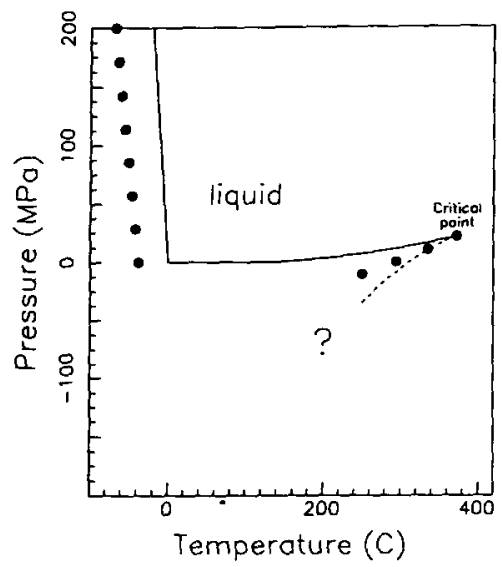

Fig. 3.

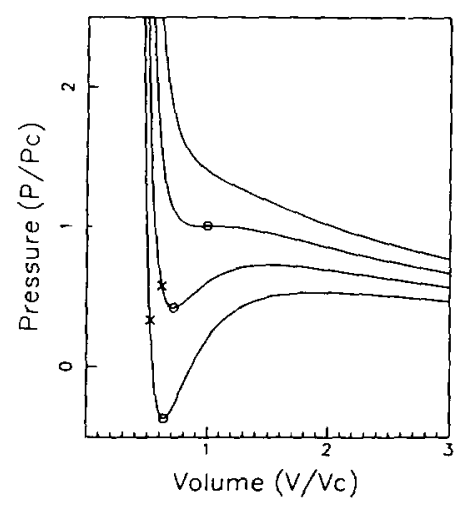

Fig. 4.

Fig. 3. Phase diagram of water: solid lines indicate the liquid-gas and the liquid-solid equilibrium boundaries. Full circles indicate the limit of homogeneous nucleation. The dashed line is the spinodal line associated with the liquid-gas critical point.

Fig. 4. Van der Waals isotherms around the critical point $\left(T_{\mathrm{c}}=1, V_{\mathrm{c}}=1, P_{\mathrm{c}}=1\right)$. The $T=1.2, T=1.0, T=0.8$ and $T=0.6$ isotherms are shown.

the lines of homogeneous nucleation.

Another important thermodynamic line is given by the limit of stability of the liquid. As this line is crossed, the system becomes unstable and any fluctuation, no matter how small, is able to drive the system into a new phase. In mean field approximation, the system is stable whenever

$$
\left(\frac{\partial^{2} A(V, T)}{\partial V^{2}}\right)_{T}=-\left(\frac{\partial P}{\partial V}\right)_{T}>0,
$$

where $A(V, T)$ is the Helmholtz potential, $V$ is the specific volume, $P$ the pressure and $T$ the temperature. In fig. 4 we show the $P$ versus $V$ isotherms for the mean field (van der Waals) case. We see that the point marked $o$ separates the stable liquid phase from the unstable phase, while the point $\mathrm{x}$ marks the equilibrium point between the gas and the liquid phases. The liquid is metastable between $x$ and o. Fig. 4 also makes clear that the van der Waals theory predicts the possibility of metastable liquid states at negative pressure. The line connecting the o points as a function of $T$ and $P$ gives information on the limit of stability of the liquid. This line, named the spinodal line, is also shown in fig. 3. On approaching the spinodal line, one observes an increase in the isothermal compressibility $K_{T}$ and in the isobaric specific heat $C_{P}$. Indeed, as shown by Compagner [5], these quantities diverge following a power-law form at the spinodal line, with exponents of -1 . 
While the gas and the liquid phases have the same symmetry, the liquid and the solid phases cannot be described by the same free energy surface and no liquid-solid second order critical point exists. However, this fact does not prevent the possibility that the liquid or the solid free energy hypersurfaces terminate in the metastable region in a spinodal line. Indeed, recent work on stretched and compressed tetrahedral crystals seems to suggest that the metastable region in solids might terminate in a spinodal instability under particular conditions [6]. In the case of liquids, this line of reasoning was pursued 17 years ago by Speedy and Angell [7]. Triggered by the observation that liquid water shows an anomalous increase in $C_{P}$ and $K_{T}$ on cooling, they postulated the existence of a thermodynamic anomaly in the free energy of the supercooled liquid. They argued that this anomalous line should run parallel to the homogeneous nucleation line on the experimentally inaccessible side. Since then, many static and dynamic quantities have been studied and shown to satisfy a power law relation at the lowest temperatures, as expected for such quantities near a spinodal [8-14]. However, theories which are not based on the existence of critical behavior in the supercooled region are also able to reproduce the experimental data with functional forms other than a power law [15].

Further important progress in this field has been made by Speedy [9] for the case of water and generalized to arbitrary fluids by Debenedetti and his colleagues [16-20]. They have observed that liquids having a line of density maxima which has a negative slope in the $P-T$ plane of their phase diagram should possess a region where the line of density maxima and the liquid spinodal line meet. Based on thermodynamic argumentation, they show that the spinodal must change slope at the point where it meets the line of density maxima. This phenomena means that the usual liquid spinodal can turn upward and be "re-entrant" from negative pressure back to positive pressure in the supercooled region of the phase diagram. This conclusion supports the possibility that the anomalies observed at positive pressure and low temperature are indeed connected with the ones observed at negative pressures. If this is the case, such a re-entrant spinodal represents a continuous limit of stability bounding the entire liquid state, preventing any continuity between amorphous ice and liquid water.

\section{The spinodal}

To test these hypotheses and to shed light on the behavior of liquid water in the metastable region, we perform an extensive set of simulations in the stable and metastable regions of the liquid state [21,22]. Computer simula- 
tions are particularly suitable for this kind of study because of the absence of any boundary and of any impurity, which in real experiments prompt heterogeneous nucleation. The limitations are given by the finite size of the simulation box and by the available computer time. At low temperatures and close to a pseudo-critical point, slowing down of the dynamics is expected. Hence we have chosen to work with a small set of particles but to perform very long equilibration and production runs.

We perform simulations consisting of systems of 216 particles enclosed in a cubic box with periodic boundary conditions. The effect of long range Coulomb forces is estimated with the reaction field method. The number of particles and size of the simulation box are fixed to give the desired specific volume, and initial equilibration to a desired temperature is achieved using Berendsen's velocity rescaling method, using a decay constant of $0.5 \mathrm{ps}$ [23]. The time step for the update of particle positions is fixed at $1.0 \mathrm{fs}$. Equilibration was considered complete when the unconstrained thermodynamic properties (pressure and potential energy) had relaxed into a steady state. We also check that particles had diffused a rms distance of at least one molecular diameter from their starting positions to avoid trapping in glassy states. Also, in almost all cases, a given simulation was started using the final particle positions of a previous simulation at as near a state point to the new one as was possible. The lengths of the runs (equilibration plus production) varied depending on the time needed to obtain reliable equilibration and measurements, but in no case were less than $250 \mathrm{ps}$. Due to the slow relaxation seen in the systems at the lowest temperatures and highest specific volumes, run times of 600 to $1000 \mathrm{ps}$ were common. By calculating the pressure for each of the temperatures and volumes studied, we have reconstructed the $P(V, T)$ surface in a large part of the supercooled and stretched regions of the phase diagram.

Because the density maximum phenomenon is tightly connected with the possibility of a re-entrant spinodal, simulations with the chosen water-water potential must exhibit, as a function of temperature, a density maximum at constant pressure or a pressure minimum at constant volume. For this reason, we first perform simulations at a fixed density $\left(1.0 \mathrm{~g} / \mathrm{cm}^{3}\right)$ as a function of temperature for different potentials commonly used to model liquid water. The resulting $P-T$ isochores are shown in fig. 5, together with the experimental data [24]. All the potentials show a pressure minimum, even if at different temperatures and pressures. Compared with the experimental density maximum, the SPC/E [25] and TIP4P [26] density maxima are shifted toward lower temperatures while the ST2 [27] density maximum is found at higher temperatures.

This apparent drawback of the ST2 potential is very useful for our numerical study. Indeed the higher temperature of the ST2 density maximum allows 


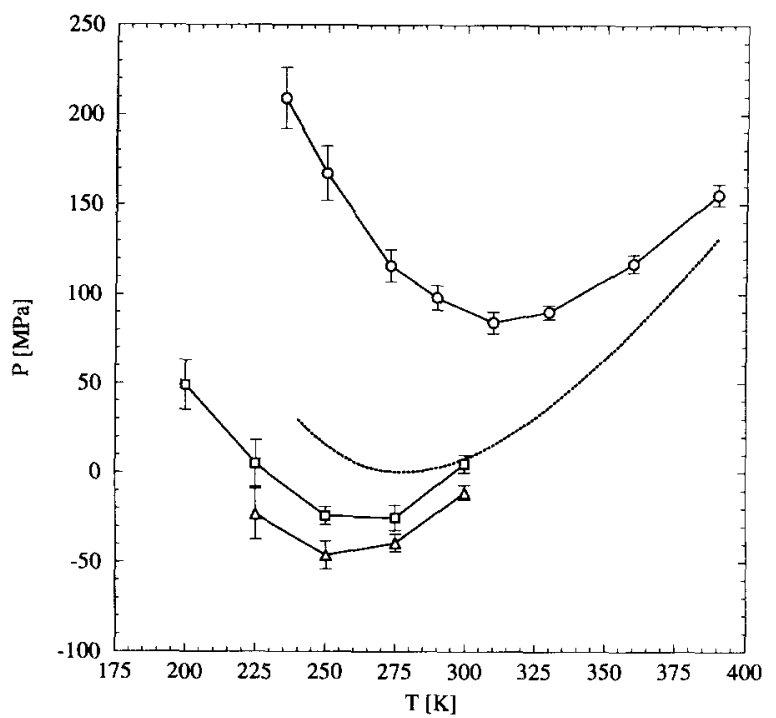

Fig. 5. Isochores at density $1.0 \mathrm{gm} / \mathrm{cm}^{3}$ from experimental data (dotted line), SPC/E potential $(\triangle)$, TIP4P potential $(\square)$, and ST2 potential (o).

equilibrium simulations of states up to $100 \mathrm{~K}$ below its TMD. Therefore, ST2 can be used to probe the thermodynamic properties and structure of the system in the region both where the liquid is strongly anomalous, and where the re-entrant spinodal is predicted to be found. For this reason, we focus our computational efforts on assemblies of ST2 particles. However, we also confirm that the results are not an artifact only of the choice of pair-potential. Hence, as indicated below, the important results obtained from ST2 simulations are confirmed in simulations using the TIP4P potential.

As we have noted above, the density maximum line if negatively sloped in the $P-T$ plane must meet the spinodal line, forcing it to become re-entrant. Because ST2 water has a negatively-sloped density maximum line at positive pressures and a monotonically decreasing spinodal, we predict that the density maximum line in ST2 water must change slope in the vicinity of the liquid spinodal. To test this prediction, we calculate the $P-T$ isochores for different densities and find for each density the temperature at which the pressure is at a minimum (fig. 7). The line of density maxima thus calculated is found to change slope around density $0.9 \mathrm{gm} / \mathrm{cm}^{3}$, avoiding the intersection with the spinodal line (shown as a full line), and the thermodynamic behavior predicted by Speedy and Debenedetti. We are led to conclude, on the basis of the data shown in fig. 7, that the liquid spinodal is not re-entrant in ST2 water $[21,22]$. This conclusion has been independently reached for ST2 by 


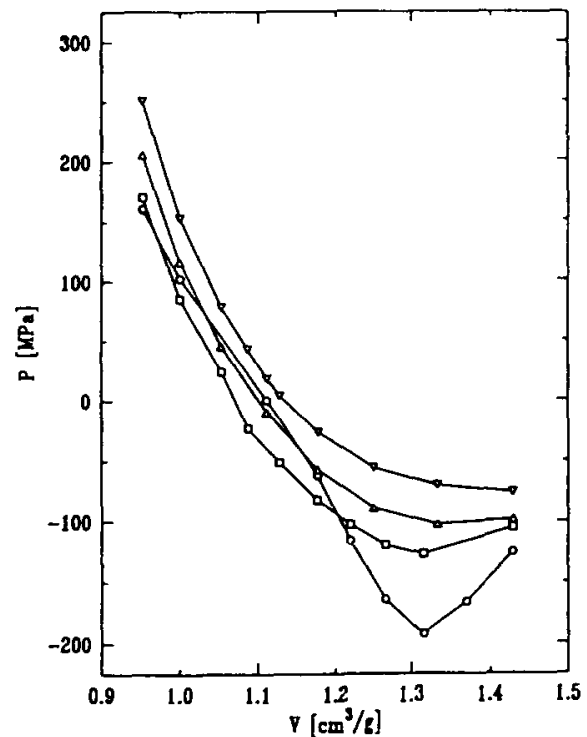

Fig. 6.

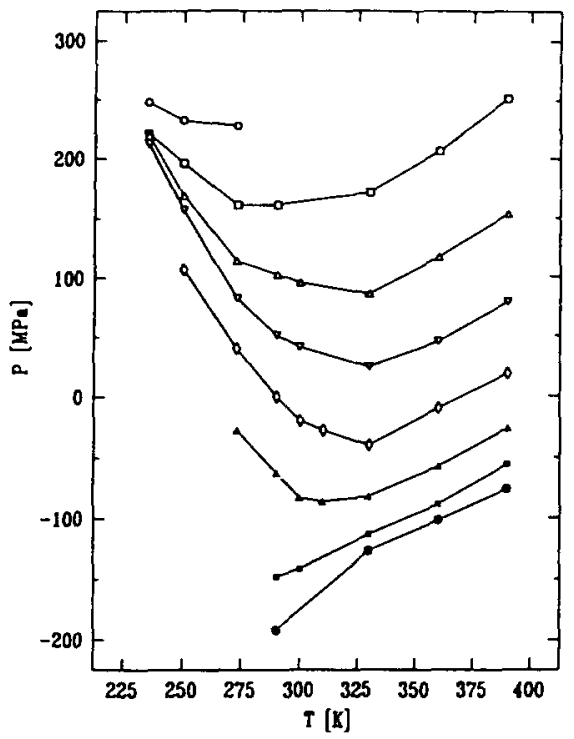

Fig. 7.

Fig. 6. ST2 $P-V$ isotherms at $T=390 \mathrm{~K}(\triangle), T=360 \mathrm{~K}(\nabla), T=330 \mathrm{~K}$ (ם) and $T=$ $290 \mathrm{~K}(0)$. This figure shows the $P-V$ isotherms at four temperatures. At all the temperatures we are able to simulate metastable states at negative pressure. The $390 \mathrm{~K}, 360 \mathrm{~K}$ and $330 \mathrm{~K}$ $P-V$ isotherms show van der Waals like behavior on stretching. The volumes and the pressures at which the system become unstable are a decreasing function of the temperature as expected in the case of normal liquids. The $290 \mathrm{~K} P-V$ isotherm crosses the $330 \mathrm{~K} P-V$ isotherm, displaying the existence of a density maximum at this temperature (i.e., a density maximum is a pressure minimum at constant volume). Interestingly enough, the spinodal pressure $P_{\mathrm{s}}$ at this temperature is lower than $P_{\mathrm{s}}$ at $T=330 \mathrm{~K}$, indicating that the spinodal line $P_{\mathrm{s}}(T)$ does not turn around but continues decreasing in pressure on lowering the temperature.

Fig. 7. ST2 isochores for different densities. From top to bottom: $1.05 \mathrm{gm} / \mathrm{cm}^{3}, 1.0 \mathrm{gm} / \mathrm{cm}^{3}$, $0.95 \mathrm{gm} / \mathrm{cm}^{3}, 0.9 \mathrm{gm} / \mathrm{cm}^{3}, 0.85 \mathrm{gm} / \mathrm{cm}^{3}, 0.8 \mathrm{gm} / \mathrm{cm}^{3}$. The full line connecting the filled circles is the spinodal line for the ST2 potential.

Striemann [28]. In our simulations using TIP4P [22], we also find that both the density maximum line changes slope in the region of $P<0$, and that the spinodal is not re-entrant.

In a separate study, we have conducted an analogous set of simulations of $\mathrm{SiO}_{2}$, as modeled by a rigid-ion Born-Mayer-Huggins (BMH) potential [29]. $\mathrm{SiO}_{2}$, both in experiments and simulations, has been shown to exhibit a density maximum similar in character to that found in water [30]. It is also a system topologically similar to water in that the liquid state is characterized by tetrahedrally coordinated species. Although the interparticle bonds in $\mathrm{SiO}_{2}$ are orders of magnitude stronger than the hydrogen bonds found in water, the thermodynamic reasoning that has been applied to predict the EOS for water 

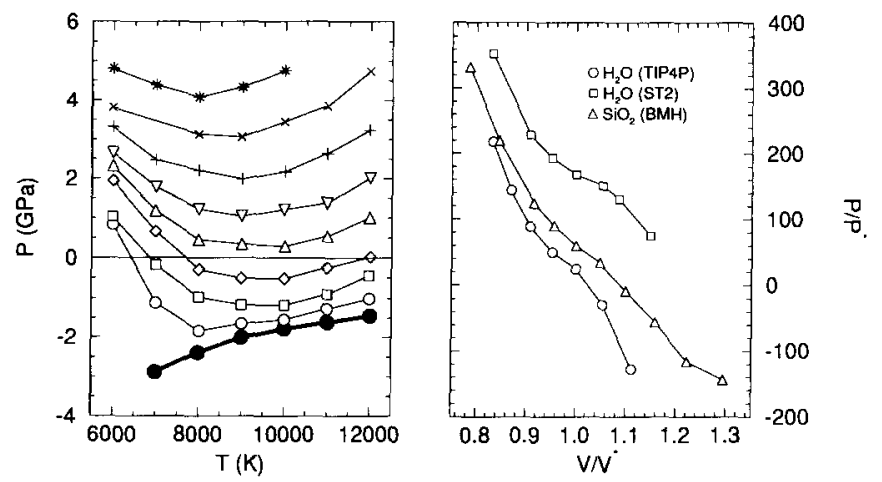

Fig. 8. (a) Liquid isochores of $P$ as a function of $T$ as calculated in simulations of $\mathrm{SiO}_{2}$ (BMH potential). The spinodal is shown as a thick line. Note that $\rho$ decreases from $2.6 \mathrm{~g} / \mathrm{cm}^{3}(*)$ to $1.9 \mathrm{~g} / \mathrm{cm}^{3}(0)$ in steps of $0.1 \mathrm{~g} / \mathrm{cm}^{3}$. (b) Representative low $T$ isotherms of $P$ as a function of $V$ for two $\mathrm{H}_{2} \mathrm{O}$ models, TIP4P $\left(T=200 \mathrm{~K}, V^{*}=1.0 \mathrm{~cm}^{3} / \mathrm{g}, P^{*}=0.5 \mathrm{MPa}\right)$ and ST2 $\left(T=250 \mathrm{~K}, V^{*}=1.0 \mathrm{~cm}^{3} / \mathrm{g}, P^{*}=1.0 \mathrm{MPa}\right)$, and for $\mathrm{SiO}_{2}$ as modeled by a rigid-ion $\mathrm{BMH}$ potential $\left(T=7000 \mathrm{~K}, V^{*}=0.455 \mathrm{~cm}^{3} / \mathrm{g}, P^{*}=20 \mathrm{MPa}\right)$.

may equally apply to $\mathrm{SiO}_{2}$. Hence, there arises the question as to whether a simulation of $\mathrm{SiO}_{2}$ would reproduce the results of ST2 and TIP4P, or perhaps instead satisfy predictions of a re-entrant spinodal.

Fig. 8a shows some preliminary results of our $\mathrm{SiO}_{2}$ simulations, in the $P-T$ plane. Though the scale of $T$ and $P$ has changed enormously, the qualitative similarity to the ST2 behavior given in fig. 7 is striking. In this regard, it is important to note the difference between the water and $\mathrm{SiO}_{2}$ potentials used: ST2 and TIP4P both model a water molecule as a rigid assembly of charged force centers, where the tetrahedral arrangement of atoms has been imposed on the intermolecular potential. The rigid-ion potential used for $\mathrm{SiO}_{2}$ however, models the system as a set of unconstrained charged soft spheres, without orientational constraints or three-body forces. The fact that these two very different potentials yield the same equation of state behavior suggests the possibility that such behavior may be a common feature in any liquid which forms a tetrahedral network.

\section{A new critical point?}

If the spinodal is not re-entrant in real water, what is the origin of the increase in $C_{P}$ and $K_{T}$ in the supercooled region? Is the ST2 potential able to reproduce this anomalous behavior with a mechanism different from that occurring when there is a re-entrant spinodal? To address these questions, we have calculated the $P-V$ isotherms at lower temperatures [21]. As shown in fig. 9, on lowering 


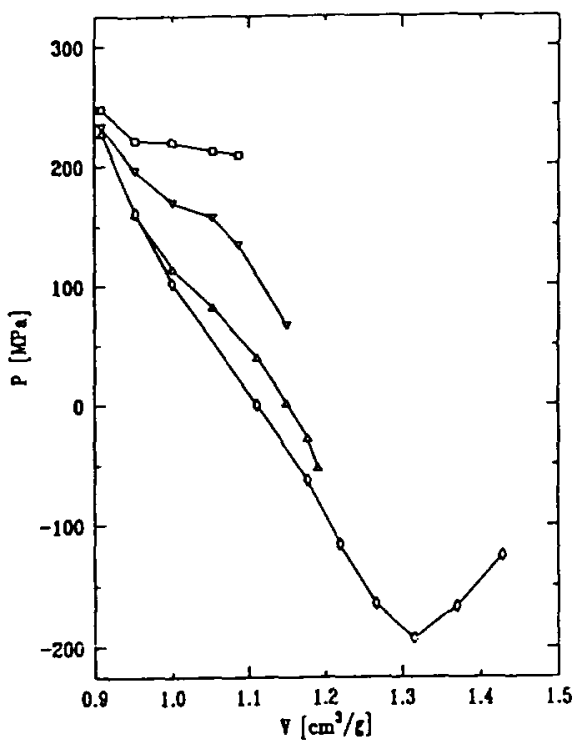

Fig. 9. ST2 $P-V$ isotherms at $T=235 \mathrm{~K}(\square), T=250 \mathrm{~K}(\triangle), \mathrm{T}=273 \mathrm{~K}(\triangle)$ and $\mathrm{T}=290$ $\mathrm{K}(\diamond)$.

the temperature the isotherms develop a stronger and stronger inflection which ends in a critical isotherm around $220 \mathrm{~K}$. The data shown in fig. 9 are indeed very similar to the van der Waals isotherm shown in fig. 4 once we remember that, being in the region with negative expansion coefficient, the pressure increases at constant volume on decreasing the temperature. ST2 water thus seems to display a novel critical point $\mathrm{c}^{\prime}$ at about $T_{\mathrm{c}^{\prime}}=220 \mathrm{~K}$ and $P_{\mathrm{c}^{\prime}}=$ $200 \mathrm{MPa}$ at a density close to $1.0 \mathrm{gm} / \mathrm{cm}^{3}$. Again, both the presence of an inflection, and the trend for it to become stronger at lower $T$ is confirmed in our TIP4P simulations [22], though the estimated critical temperature is a few tens of degrees $\mathrm{K}$ lower in this system. Notably, our simulations of $\mathrm{SiO}_{2}$ also show inflections in the isotherms of $P$ versus $V$ for the low $T$ liquid: see fig. 8b. Thus, again, it appears that these equation of state features may be generic to many tetrahedral liquids, rather than a peculiarity of a particular pair-potential.

If this critical point exists then we should be able to identify two phases with the same symmetry and a coexistence line between them. To investigate the structural difference between the two phases we study the structure of the liquid at a temperature close to but lower than $T_{\mathrm{c}^{\prime}}$ at two densities far from the critical density. This is the same as looking at the structure of a liquid-gas system along a supercritical isotherm for densities respectively smaller and larger than the liquid-gas critical density. In this case the high density structure 


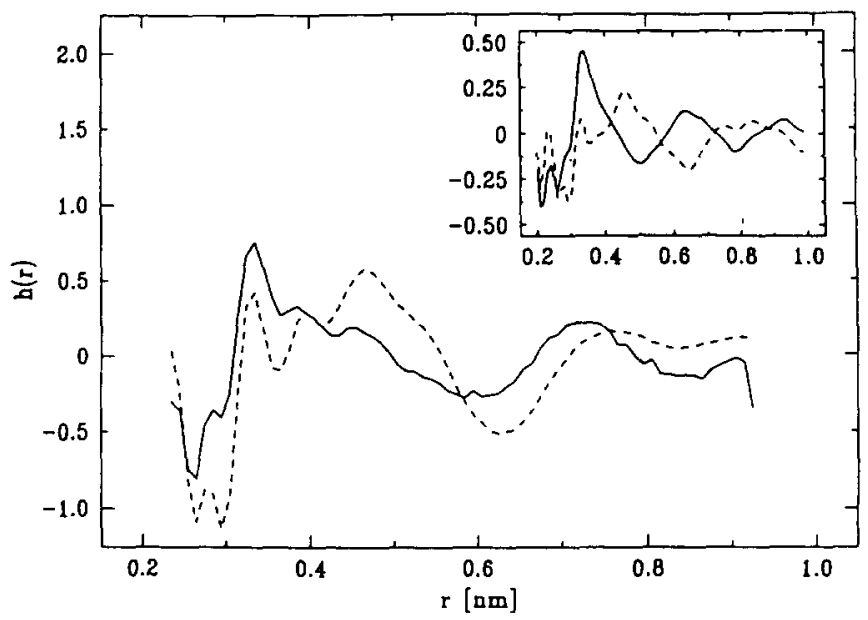

Fig. 10. Neutron-averaged Fourier transform of the structure factor $h(r)$. Dashed line: $\rho=0.92 \mathrm{gm} / \mathrm{cm}^{3}$; full line: $\rho=1.05 \mathrm{gm} / \mathrm{cm}^{3}$. In the inset, the experimentally measured $h(r)$ functions for the low density amorphous ice (dashed line) and the high density amorphous ice (full line) from ref. [24] are shown.

would resemble the liquid structure, while the low density structure would resemble the one characteristic of the gas phase. The comparison between the two ST2 structures is shown in fig. 10, where we show the $h(r)$ function, i.e., the neutron-averaged Fourier transform of the structure factor, for density $0.92 \mathrm{gm} / \mathrm{cm}^{3}$ and $1.05 \mathrm{gm} / \mathrm{cm}^{3}$ at $T=235 \mathrm{~K}$. The difference between the two structures strikingly resembles the difference observed experimentally between the structures of high density amorphous (HDA) and low density amorphous (LDA) ice [31,32].

Then, in ST2 water, for pressure lower than $200 \mathrm{MPa}$, it is possible to find completely reversible paths continuously connecting states where the structure of the liquid is similar to the HDA and to the LDA structure. In the same range of pressure, a completely reversible path connects the low temperature glassy state to the normal liquid.

As is well known from the theory of critical phenomena [33], the presence of a critical point affects the response functions in an area around the critical point; as seen in fig. 9, the supercritical isotherms are affected by the existence of the critical point. Along paths in the $P-T$ plane in the one-phase region (i.e. on lowering the temperature at constant pressure) one expects maxima in $C_{P}$ and in $K_{T}$ because the response functions increase on approaching the critical point and decrease on getting further away.

Are the experimentally-observed increases in $C_{P}$ and $K_{T}$ related to the existence of an unreachable critical point? To test this possibility we compare $C_{P}$ 


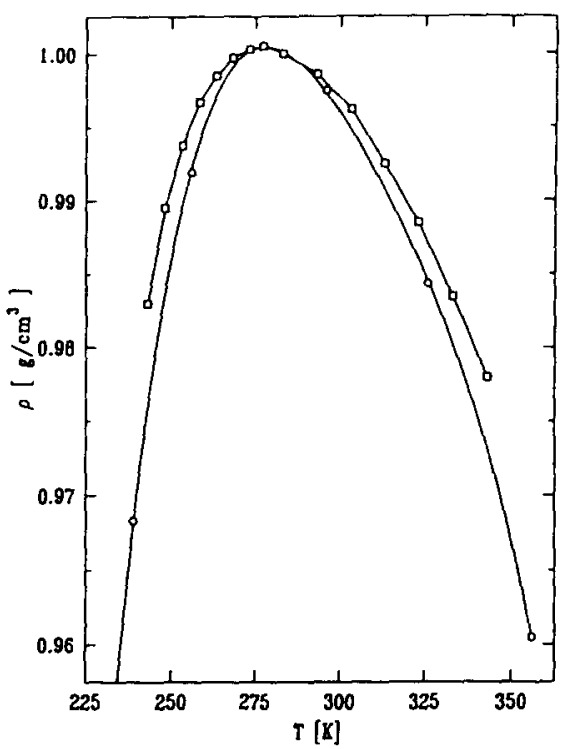

Fig. 11. Temperature dependence of the density at atmospheric pressure in water (full line) and simulated ST2 water (dashed line).

and $K_{T}$ calculated from our simulations with the experimentally measured $C_{P}$ and $K_{T}$. We compare the experimental data for each temperature and pressure with the corresponding ST2 data at temperature $34 \mathrm{~K}$ higher and a pressure $95 \mathrm{MPa}$ higher to take into account the different positions of the density maxima in the $P-T$ plane. To support the validity of this procedure, we show in fig. 11 the comparison of the experimental density at atmospheric pressure with the shifted ST2 data. The agreement is rather good. Using the same shift we compare in fig. $12 C_{P}$ and $K_{T}$. Again remarkable agreement is observed, both qualitatively and quantitatively.

The agreement between the experimental data and the ST2 shifted data appears to validate the description of the liquid state offered by our ST2 calculations, and as confirmed in TIP4P. However, the results shown here must be submitted to extensive tests, particularly using much larger systems. We have begun this task, by confirming the properties found in the vicinity of the spinodal of the 216 molecule ST2 system, in simulations employing 1728 molecules [22]. However, more tests of this nature need to be done close to the region of new critical phenomena that we propose. The importance of this task argues for a strong effort in implementing MD programs for waterlike potentials on new powerful parallel computers. Analytical work on simple potentials showing a density maximum should also be pursued; already such approaches have revealed a rich set of possible behaviors [16-20,34-37]. 

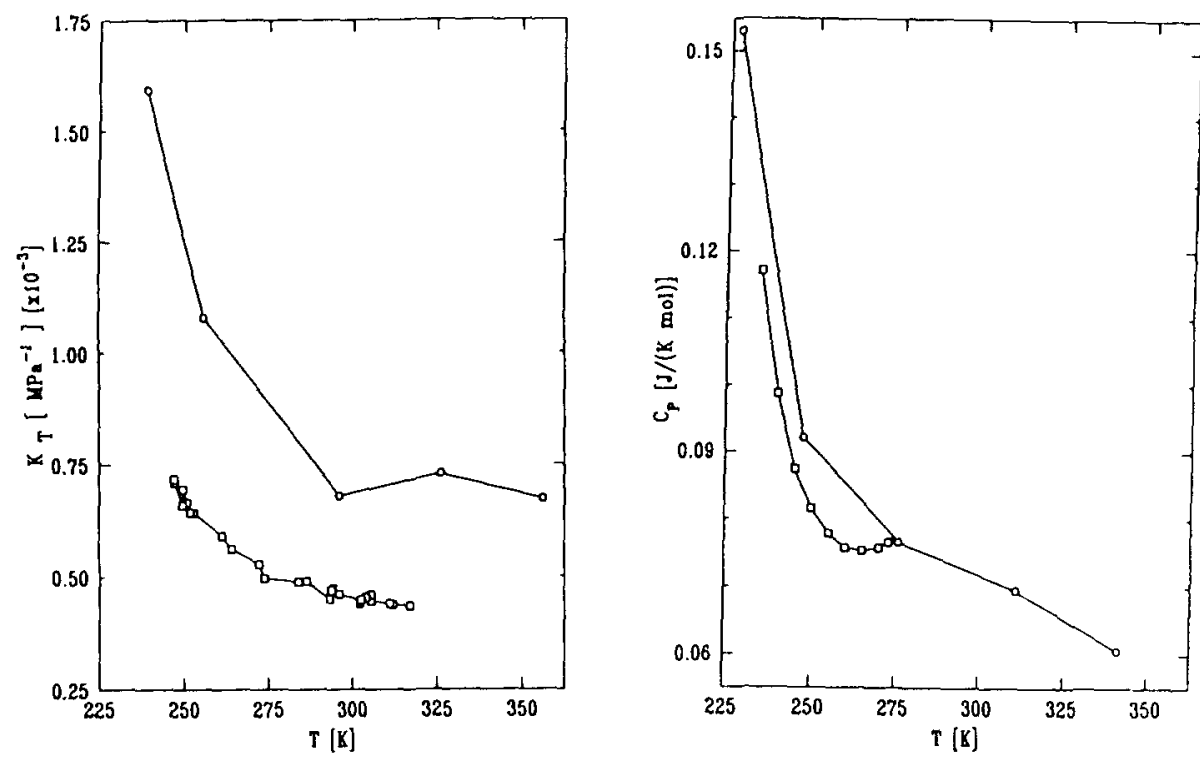

Fig. 12. (a) Temperature dependence of $K_{T}$ at atmospheric pressure in water ( $\square$ ) and simulated ST2 water (o). (b) Temperature dependence of $C_{P}$ at atmospheric pressure in water ( $\square$ ) and simulated ST2 water (0).

If our data are confirmed by these extensive checks, then it will become important to perform experiments as close as possible to the new critical point, which according to the $P$ and $T$ shift used above, should be located in real water at $T=185 \mathrm{~K}$ and $P=120 \mathrm{~Pa}$. At the same time, we hope that measurements in the stretched region can be extended to search for the change in slope of the line of density maxima [38].

\section{Amorphous solid water}

Evidence for the occurrence of a critical point in supercooled water may also be found in the behavior of amorphous solid water. Though amorphous solid water may be prepared by a number of different procedures [39], in general it appears that one of LDA or HDA ice is the result. LDA ice has been observed to abruptly transform into HDA ice when isothermally compressed at $77 \mathrm{~K}$, in a process described as an "apparently first-order phase transition" [40].

To investigate a possible connection between the apparent phase transition observed in amorphous solid water, and the occurrence of a critical point in supercooled liquid water, we use computer simulations to study the LDA $\rightarrow$ HDA ice transformation over a wider range of $T$ than has been attempted in exper- 
iments ${ }^{\# 1}$ [41]. We first prepare a LDA ice configuration by quenching a liquid state configuration to low $T$, and then isothermally compress it. Fig. 13 shows the results for this process for both ST2 and TIP4P, both of which display a region of $P$ over which $\rho$ changes abruptly, qualitatively reproducing the LDA $\rightarrow$ HDA ice transition. Furthermore, if we decompress the resulting high density phase through $P=0$, and into the region of hydrostatic tension, the results indicate that the reverse HDA $\rightarrow$ LDA ice transition occurs. The fact that both the forward and reverse transformations may be observed is consistent with the interpretation that LDA and HDA ice are separated in the $P$ $T$ plane by a line of first-order phase transitions. As described in detail elsewhere [41], the observation that the forward and reverse transitions do not occur at the same $P$ is a reflection of the suppression of nucleation kinetics in this low $T$ region where molecular mobility is strongly hindered.

The compression/decompression cycles at different $T$ in fig. 13 indicate that the observed size of the hysteresis loop decreases as $T$ increases [41]. This behavior is consistent with the possibility that the line of first-order transitions separating LDA and HDA ice comes to an end near the low $T$ limit of the region where the supercooled liquid is observed. A second-order critical point is typically found at the termination of a line of first-order phase transitions, and its existence is consistent with that of the critical point inferred to exist from the liquid state data.

Given the calculated thermodynamic behavior discussed thus far, what kind of phase diagram arises for water in the supercooled and stretched regions? The phase diagram we propose (see fig. 14) postulates the existence of a line of first order phase transitions that separates LDA and HDA ice. The observed properties of amorphous ice can be understood through a consideration of the behavior of the limits of metastability (also spinodals) that must be associated with a first order phase transition line ending in a critical point. These two new spinodal lines, distinct from the liquid spinodal examined above, and denoted by $\mathrm{L}$ and $\mathrm{H}$ in fig. 14, define the absolute limits of thermal and mechanical stability for the two phases. The line L is the metastability limit for LDA ice, while $\mathrm{H}$ defines the metastability limit for HDA ice. Hence, LDA ice is the more stable amorphous solid below the first order transition line F, yet may also be observed in the region above $F$ but below $L$, where it is metastable with respect to HDA ice. Above L, LDA ice becomes unstable. Similarly, HDA ice is the more stable form above $F$, is metastable with respect to LDA ice between $\mathrm{F}$ and $\mathrm{H}$, and is unstable below $\mathrm{H}$. The critical point that terminates

\#1 In this regard, we were motivated by the successful observation in computer simulation of the transition from ice $I_{h}$ to HDA ice, see [42]. 


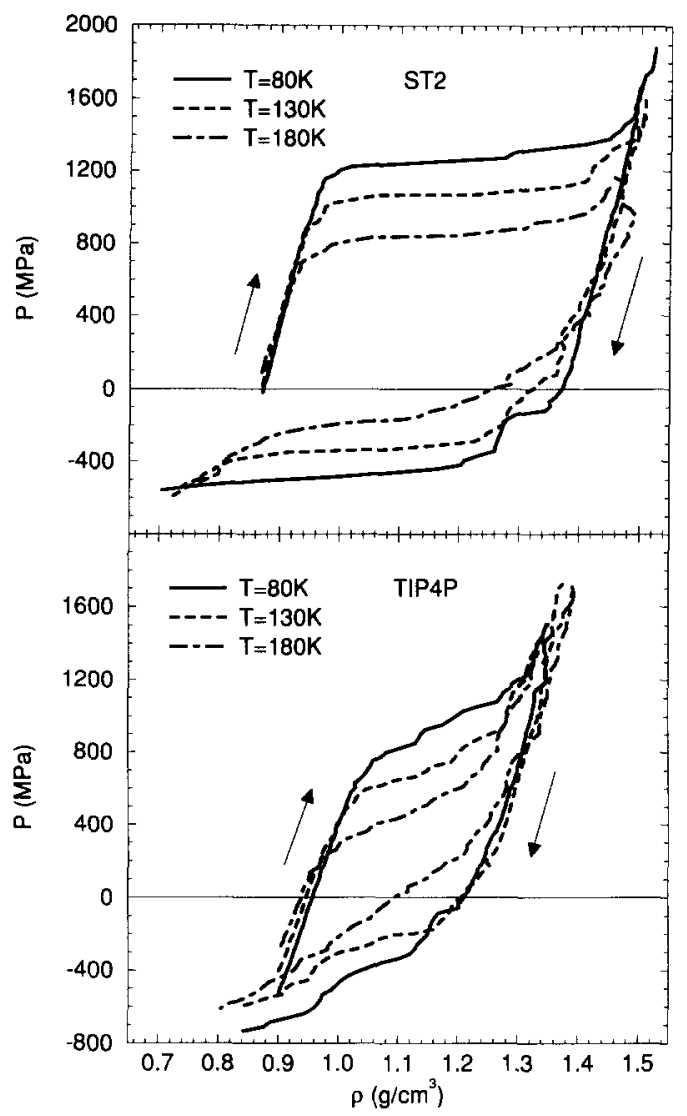

Fig. 13. Response of the density $\rho$ during isothermal compression (up-arrows) and decompression (down-arrows) of amorphous solid water simulated with ST2 (top panel) and TIP4P (bottom panel), at several different $T$. The systems fracture at the points of lowest $P$ and $\rho$ shown along the decompression paths.

the line of first order phase transitions itself accounts for the thermodynamic anomalies observed in both supercooled and stable liquid water.

\section{Conclusion}

In summary, the ST2 and TIP4P data show no re-entrant spinodal at negative pressure. They do show a novel critical point at low temperature and high pressure, which appears to be related to a transition between two structures that we have shown to be similar to the HDA and LDA structures. Also the data do not predict any "real" critical behavior in $K_{T}$ or $C_{P}$ at atmospheric 


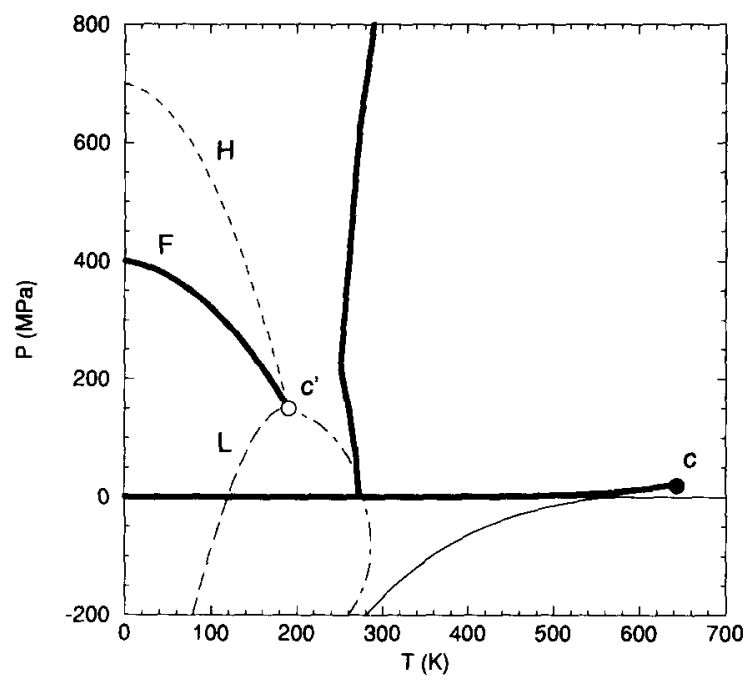

Fig. 14. A schematic phase diagram for water, incorporating the features proposed in the text so as to account for experimental observations. The equilibrium solid-liquid, solid-gas and liquid-gas phase transitions are shown as heavy lines. The liquid-gas transition line ends in the liquid-gas critical point (•). Metastable equilibrium features for the extension of the liquid state phase diagram into the supercooled and stretched regions are also shown: the liquid spinodal (thin solid line) is monotonic in $T$ and does not intersect the TMD line (dot-dashed line), which itself changes slope at $P<0$. A line of first-order phase transitions $F$ separates LDA and HDA ice, and ends in a critical point (o). Two spinodals, L (long-dashed) and $H$ (short-dashed) must occur above and below the LDA/HDA ice transition line, and their locations determine the observed transformations of amorphous solid water.

pressure, but rather a maximum in $K_{T}$ and $C_{P}$ for pressures lower than the critical pressure. The high density structure (defective tetrahedral network) that smoothly transforms into the low density structure (random tetrahedral network) on lowering the temperature is reminiscent of the progressive growth of four-bonded clusters implied by the model of Stanley and Teixeira [15]. The temperature at which $K_{T}$ is maximum, which indicates the point of smooth transition between the two structures in the ST2 system, is similar to the percolation point of low density tetrahedrally coordinated molecules in the Stanley-Teixeira model.

The data also support the possibility of a continuity of states connecting liquid water with the amorphous substance obtained experimentally by hyperquenching small liquid droplets or by vapor deposition. On a slow cooling of the liquid, the glass transition is prevented by the homogeneous nucleation event. Indeed, since the growing cluster has a structure with a local order roughly similar to the ice structure, the probability that one of these clusters will have the correct shape (topology) and size to irreversibly transform the 
system into the solid state is large. The simulation data also suggest that the amorphous substance that one obtains at low temperature and atmospheric pressure is, if truly in metastable equilibrium, on the same free energy surface as the liquid state. From an experimental point of view the situation is still unclear. The possibility of a continuity of states between amorphous and liquid phases [43,44] as well as the opposite behavior [45] has been suggested on the basis of different thermodynamic arguments based on measured values of $C_{P}$ and estimated entropies. A recent analysis [46], however, indicates that a "fragile-to-strong" liquid transformation occurs during hyperquenching of liquid water. Such a phenomenon is consistent with the passage of the (fragile) equilibrium liquid through the neighborhood of the critical point into the region dominated by the (presumably strong) LDA ice. Molecular dynamics simulations, where the nucleation event can be bypassed by the cleanness of the sample and by the finite size, should be used to refute or confirm these arguments.

\section{Acknowledgements}

We wish to thank M.-C. Bellissent-Funel, A. Geiger, S. Sastry, and J. Teixeira for helpful interactions throughout the course of this work. We also thank $\mathrm{L}$. Striemann for kindly providing us with MD code and with his data prior to publication. This work was supported by grants from BP and NSF.

\section{References}

[1] F. Sciortino, A. Geiger and H.E. Stanley, Nature 354 (1991) 218; J. Chem. Phys. 96 (1992) 3857.

[2] C.A. Angell, Ann. Rev. Phys. Chem. 34 (1983) 593;

C.A. Angell, in: Water: A Comprehensive Treatise, F. Franks, ed. (Plenum, New York, 1982) ch. 1.

[3] E.W. Lang and H.-D. Lüdemann, Angew. Chem. Int. Ed. Engl. 21 (1982) 315.

[4] A. Geiger and H.E. Stanley, Phys. Rev. Lett. 49 (1982) 1749; H.E. Stanley, J. Teixeira, A. Geiger and R.L. Blumberg, Physica A 106 (1981) 260.

[5] A. Compagner, Physica 72 (1974) 115.

[6] F. Sciortino, H.E. Stanley, J. Shao, G.H. Wolf, M. Hemmati and C.A. Angell, Science, submitted.

[7] R.J. Speedy and C.A. Angell, J. Chem. Phys. 65 (1976) 851.

[8] M. Oguni and C.A. Angell, J. Chem. Phys. 78 (1983) 7334.

[9] R.J. Speedy, J. Phys. Chem. 86 (1982) 982.

[10] R.J. Speedy, J. Phys. Chem. 86 (1982) 3002.

[11] R.J. Speedy, J. Phys. Chem. 91 (1987) 3354.

[12] D.E. Hare and C.M. Sorensen, J. Chem. Phys. 87 (1987) 4840. 
[13] F.X. Prielmeier, E.W. Lang, R.J. Speedy and H.-D. Lüdemann, Phys. Rev. Lett. 59 (1987) 1128.

[14] F.X. Prielmeier, E.W. Lang, R.J. Speedy and H.-D. Lüdemann, Ber. Bunsenges. Phys. Chem. 92 (1988) 1111.

[15] H.E. Stanley and J. Teixeira, J. Chem. Phys. 73 (1980) 3404.

[16] P.G. Debenedetti and M.C. D'Antonio, J. Chem. Phys. 84 (1986) 3339.

[17] P.G. Debenedetti and M.C. D'Antonio, J. Chem. Phys. 85 (1986) 4005.

[18] M.C. D'Antonio and P.G. Debenedetti, J. Chem. Phys. 86 (1987) 2229.

[19] P.G. Debenedetti and M.C. D'Antonio, AIChE J. 34 (1988) 447.

[20] P.G. Debenedetti, V.S. Raghaven and S.S. Borick, J. Phys. Chem. 95 (1991) 4540.

[21] P.H. Poole, F. Sciortino, U. Essmann and H.E. Stanley, Nature 360 (1992) 324; preprint.

[22] P.H. Poole, F. Sciortino, U. Essmann and H.E. Stanley, Phys. Rev. E 48 (1993) 3799.

[23] H.J.C. Berendsen, J.P.M. Postma, W.F. van Gunsteren, A. DiNola and J.R. Haak, J. Chem. Phys. 81 (1984) 3684.

[24] L. Haar, J.S. Gallagher and G.S. Kell, in: National Bureau of Standards - National Research Council Steam Tables (McGraw-Hill, New York, 1985).

[25] H.J.C. Berendsen, J.R. Grigera and T.P. Straatsma, J. Phys. Chem. 91 (1987) 6269.

[26] W.L. Jorgensen, J. Chandrasekhar, J. Madura, R. W. Impey and M. Klein, J. Chem. Phys. 79 (1983) 926.

[27] F.H. Stillinger and A. Rahman, J. Chem. Phys. 60 (1974) 1545.

[28] L. Striemann, Ph.D. Thesis (Univ. of Dortmund, 1992).

[29] L.V. Woodcock, C.A. Angell and P. Cheeseman, J. Chem. Phys. 65 (1976) 1565.

[30] C.A. Angell and H. Kanno, Science 193 (1976) 1121;

C.A. Angell, P.A. Cheeseman and C.C. Phifer, Mat. Res. Soc. Symp. Proc. 63 (1985) 85;

D.R. Perchak and J. M. O'Reilly, J. Non-Cryst. Solids, in press.

[31] M.R. Chowdhury, J.C. Dore and J.T. Wenzel, J. Non-Cryst. Solids 53 (1982) 247.

[32] M.-C. Bellisent-Funel, J. Teixeira and L. Bosio, J. Chem. Phys. 87 (1987) 2231.

[33] H.E. Stanley, Introduction to Phase Transitions and Critical Phenomena (Oxford Univ. Press, New York, 1971).

[34] P.C. Hemmer and G. Stell, Phys. Rev. Lett. 24 (1970) 1284.

[35] G. Stell and P.C. Hemmer, J. Chem. Phys. 56 (1972) 4274.

[36] F.H. Stillinger and T.A. Weber, J. Chem. Phys. 68 (1978) 3837.

[37] S. Sastry, F. Sciortino and H.E. Stanley, J. Chem. Phys. 98 (1993) 9863; Chem. Phys. Lett. 207 (1993) 275.

[38] J.L. Green, D.J. Durben, G.H. Wolf and C.A. Angell, Science 249 (1990) R649.

[39] E.F Burton and W.F. Oliver, Nature 135 (1935) 505; Proc. R. Soc. London A153 (1935) 166 ;

P. Brügeller and E. Mayer, Nature 288 (1980) 569;

J. Dubochet and A. W. McDowall, J. Microscopy 124 (1981) RP3;

E. Mayer and P. Brüggeller, Nature 298 (1982) 715;

O. Mishima, L.D. Calvert and E. Whalley, Nature 310 (1984) 393;

E. Mayer, J. Appl. Phys. 58 (1985) 663;

G.P. Johari, A. Hallbrucker and E. Mayer, Nature 330 (1987) 552.

[40] O. Mishima, L.D. Calvert and E. Whalley, Nature 314 (1985) 76.

[41] P.H. Poole, U. Essmann, F. Sciortino and H.E. Stanley, Phys. Rev. E 48 (1993) 4605.

[42] J.S. Tse and M.L. Klein, Phys. Rev. Lett. 58 (1987) 1672; J. Chem. Phys. 92 (1990) 3992.

[43] A. Hallbrucker, E. Mayer and G.P. Johari, Phil. Mag. 60 (1989) 179.

[44] E. Whalley, D.D. Klug and Y.P. Handa, Nature 342 (1989) 782.

[45] R.J. Speedy, J. Phys. Chem. 96 (1992) 2322.

[46] C.A. Angell, J. Phys. Chem. 97 (1993) 6339. 